\title{
$\gamma$
}

$$
\begin{array}{r}
D O E / S S D C-76-45 / 8 \\
\text { SSDC-8 } \\
\text { Revision } 1
\end{array}
$$

\section{Standardization Guide for Construction and Use of MORT-Type Analytic Trees \\ February 1992}
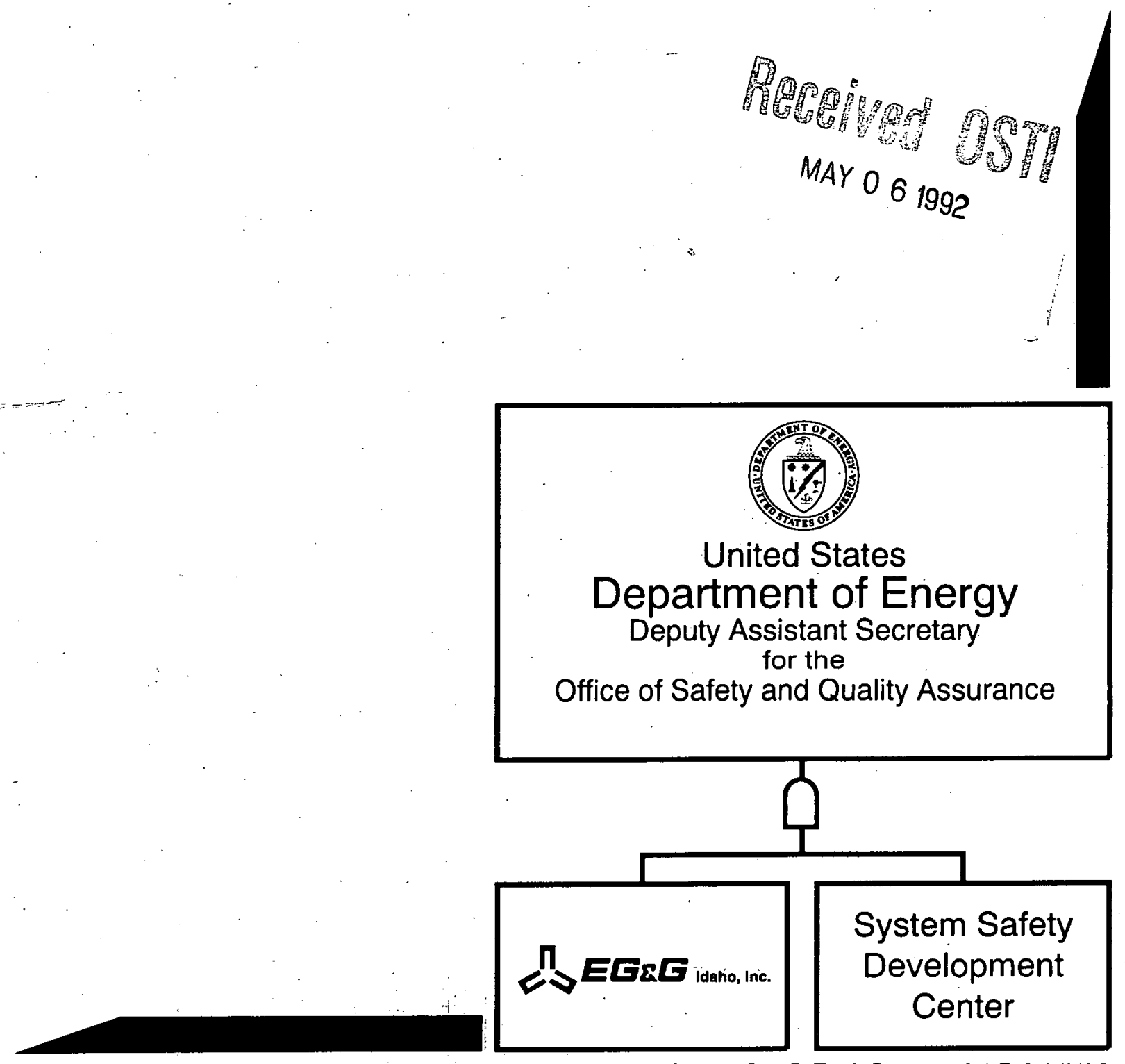

WORK PERFORMED UNDER DOE CONTRACT NO. DE-ACO7-761D01570

DISTRIBUTION OF THIS DOCUMENT IS UNLIMITED 


\section{DISCLAIMER}

This report was prepared as an account of work sponsored by an agency of the United States Government. Neither the United States Government nor any agency Thereof, nor any of their employees, makes any warranty, express or implied, or assumes any legal liability or responsibility for the accuracy, completeness, or usefulness of any information, apparatus, product, or process disclosed, or represents that its use would not infringe privately owned rights. Reference herein to any specific commercial product, process, or service by trade name, trademark, manufacturer, or otherwise does not necessarily constitute or imply its endorsement, recommendation, or favoring by the United States Government or any agency thereof. The views and opinions of authors expressed herein do not necessarily state or reflect those of the United States Government or any agency thereof. 


\section{DISCLAIMER}

Portions of this document may be illegible in electronic image products. Images are produced from the best available original document. 


\section{DISCLAIMER}

This report was prepared as an account of work sponsored by the United States Government. Neither the United States nor the United States Department of Energy nor any of their employees, nor any of their contractors, subcontractors, or their employees, makes any warranty, expressed or implied, or assumes any legal liability or responsibility for the accuracy, completeness, or usefulness of any information, apparatus, product or process disclosed, or represents that its use would not infringe privately owned rights.

This document is intended to be in compliance with the command literature as of the indicated date of issue. The user must verify the state of compliance with current United States Department of Energy command literature.

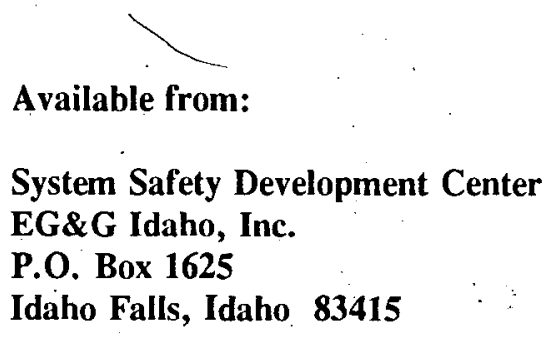


DOE/SSDC-76-45/8-Rev.1

DE92 013784

\section{Standardization Guide for Construction and Use of MORT-Type Analytic Trees}

J. R. Buys

Published February 1992

System Safety Development Center Idaho National Engineering Laboratory EG\&G Idaho, Inc. Idaho Falls, Idaho 83415

Prepared for the U.S. Department of Energy Office of Safety and Quality Assurance Under DOE Idaho Field Office Contract No. DE-AC07-76ID01570 


\section{ACKNOWLEDGMENTS}

Special acknowledgment is extended to the staff of the System Safety Development Center and to reviewers throughout the DOE complex for their helpful suggestions and guidance.

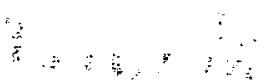




\section{CONTENTS}

ACKNOWLEDGMENTS $\ldots \ldots \ldots \ldots \ldots \ldots \ldots \ldots \ldots \ldots \ldots \ldots \ldots$ iii

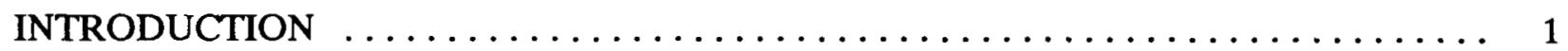

ANALYTIC TREES $\ldots \ldots \ldots \ldots \ldots \ldots \ldots \ldots \ldots \ldots \ldots \ldots \ldots \ldots \ldots \ldots \ldots$

SUMMARY $\ldots \ldots \ldots \ldots \ldots \ldots \ldots \ldots \ldots \ldots \ldots \ldots \ldots \ldots \ldots \ldots \ldots \ldots \ldots$

Analysis Steps for Analytic Tree Use $\ldots \ldots \ldots \ldots \ldots \ldots \ldots \ldots \ldots \ldots \ldots$

Analytic Tree Construction Principles $\ldots \ldots \ldots \ldots \ldots \ldots \ldots \ldots \ldots \ldots \ldots \ldots$

ANALYSIS STEPS $\ldots \ldots \ldots \ldots \ldots \ldots \ldots \ldots \ldots \ldots \ldots \ldots \ldots \ldots \ldots \ldots \ldots$

TREE CONSTRUCTION $\ldots \ldots \ldots \ldots \ldots \ldots \ldots \ldots \ldots \ldots \ldots \ldots \ldots \ldots$

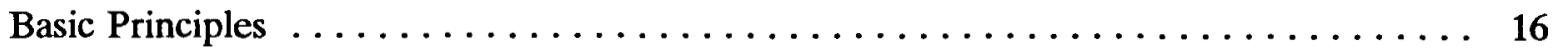

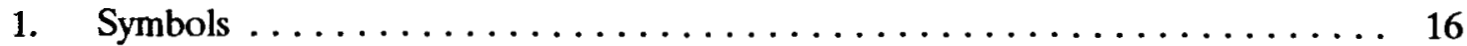

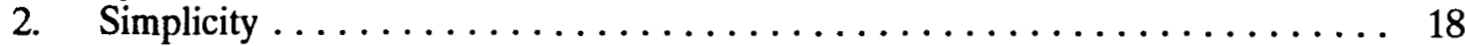

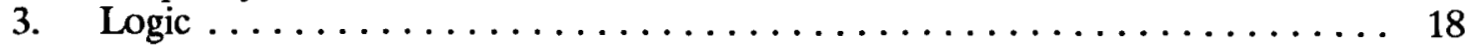

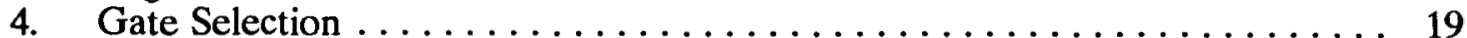

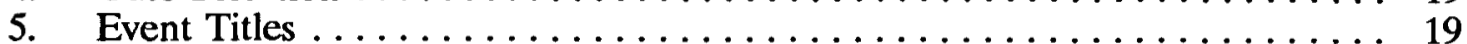

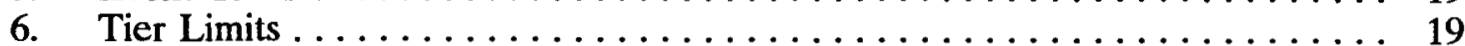

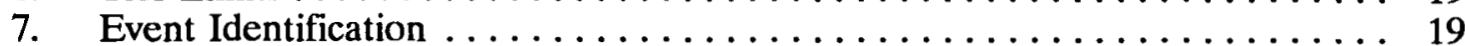

8. Modified Event Identification $\ldots \ldots \ldots \ldots \ldots \ldots \ldots \ldots \ldots \ldots \ldots \ldots \ldots \ldots$

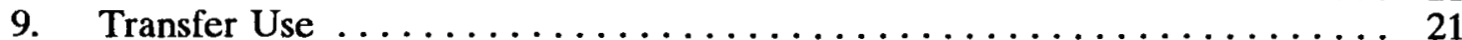

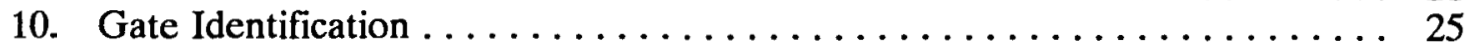

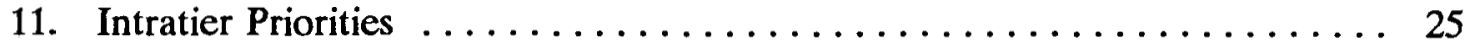



COMPLETED SSDC PUBLICATIONS $\ldots \ldots \ldots \ldots \ldots \ldots \ldots \ldots \ldots \ldots \ldots$

\section{FIGURES}

1. Some Practical Applications of Analytical Trees. ................. 3

2. Cross Index of Event and Logic Symbols. $\ldots \ldots \ldots \ldots \ldots \ldots \ldots \ldots \ldots \ldots \ldots \ldots$

3. Analytic Tree EVENT Symbols. $\ldots \ldots \ldots \ldots \ldots \ldots \ldots \ldots \ldots \ldots \ldots \ldots \ldots \ldots \ldots$



5. Analytic Tree TRANSFER Symbols. . . . . . . . . . . . . . . . . 8

6. Acceptable Tier Arrangements. . . . . . . . . . . . . . . .

7. Examples of Good and Poor Logic. . . . . . . . . . . . . . . . . . 10

8. Sample Analytic Tree - Sheet $1 . \ldots \ldots \ldots \ldots \ldots \ldots \ldots \ldots \ldots \ldots \ldots \ldots \ldots \ldots \ldots$ 
9. Sample Analytic Tree - Sheet $2 . \ldots \ldots \ldots \ldots \ldots \ldots \ldots \ldots \ldots \ldots \ldots \ldots \ldots \ldots$

10. Another Numeric Progression in Tree Format. ................. 22

11. Alphanumeric Progression from the Fourth Subtier to the TRANSFER in Tree Format.

\section{TABLES}

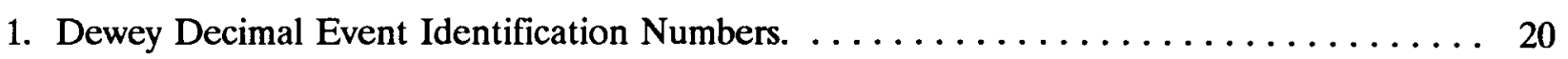

2. Modified Dewey Decimal Event Identification Numbers. . . . . . . . . . . 23 


\section{Standardization Guide for Construction and Use of MORT-Type Analytic Trees}

\section{INTRODUCTION}

Since the introduction of MORT (Management Oversight and Risk Tree) technology as a tool for evaluating the success or failure of safety management systems, there has been a proliferation of analytic trees throughout DOE and its contractor organizations. Standard "fault tree" symbols have generally been used in logic diagram or tree construction, but new or revised symbols have also been adopted by various analysts. Additionally, a variety of numbering systems have been used for event identification. The consequent lack of standardization has caused some difficulties in interpreting the trees and following their logic.

This guide seeks to correct this problem by providing a standardized system for construction and use of analytic trees. Future publications of the DOE System Safety Development Center (SSDC) will adhere to this guide. It is recommended that other DOE organizations and contractors also adopt this system to achieve intra-DOE uniformity in analytic tree construction.

The proposed system is the outgrowth of the SSDC's experience in developing and teaching the use and construction of MORT diagrams and other analytic trees. It is equally applicable to both "success," "positive," or "objective" trees and "failure," "fault," or "negative" trees. 


\section{ANALYTIC TREES}

The use of analytic trees originated as "fault tree analysis" during the early 1960s in the aerospace industry, as an attempt to prevent oversights, particularly at system interfaces, which had previously resulted in costly retrofits or inordinately short operational lifetimes for promising systems. Fault tree analysis was strongly hardware-oriented, but also showed promise as an analytic tool for evaluation of systems involving a great deal of human performance. Development of the MORT concept a decade later, and its subsequent acceptance by DOE for agency-wide use, made application of the analytical tree techniques to management systems a reality.

An analytic tree is simply a graphical display of information to aid the user in conducting a deductive analysis of any system (human, hardware, or environmental) to determine critical paths to success or failure. It identifies the details and interrelationships that must be considered to prevent oversights or omissions that lead to failures. It enables the analyst to:

1. Systematically identify the possible paths from base events to predicted outcome

2. Display a clear visual record of the analytical process

3. Identify management system weaknesses and strengths

4. Provide a basis for rational decisionmaking by management.

In an analytic tree, a top or major event or outcome is stated. It may be either a desired objective or goal, or an unwanted or injurious occurrence. On the next lower tier are listed those events required to achieve the top event. Each of these is subsequently broken down into its constituents to reveal the events, causes, and sources that contribute to the occurrence of the top event. Construction of an analytic tree, therefore, constitutes a deductive analysis of a management system or safety system, proceeding from general to specific, or outcome to source, and answering the question, "How could this happen?".

Once an analytic tree has been developed, it can be used as a tool to aid in achievement of "first-time-safe" operations; assurance of successful completion of a desired objective; prevention of significant accidents by foreseeing and avoiding managerial and operational oversights and omissions; and maintenance of effective total loss control. The analytic tree can also be used after-the-fact in investigation of injuries, property damage, programmatic degradation, etc., to identify not just the symptoms, but also the root causes and sources of these accidents and the management system weaknesses that permitted them to occur (Figure 1). 


\section{"Positive"}

"Success"

or

"Objective"

Trees
"Negative"

or
"Fault"
Trees

- Accident/Incident

Investigation

- Achieving and maintaining a state of operational readiness

- Accomplishment of specific goals and objectives
- Probabilistic Risk

Assessment

- System Appraisal/

Assessment

- Root Cause Analysis

- Reliability/Fault Analysis

Figure 1. Some Practical Applications of Analytical Trees. 


\section{SUMMARY}

In the following sections of this monograph, analytic tree analysis steps and construction principles will be discussed. Both are summarized here to provide the user with a tool for rapid preview and a checklist for analytic tree-based system analysis.

\section{Analysis Steps for Analytic Tree Use}

1. Define the top event

2. Acquire a working knowledge of the system to be analyzed

3. Construct the analytic tree

4. Validate the analytic tree

5. Evaluate the analytic tree

6. Conduct tradeoff studies (risk/benefit studies)

7. Provide management with the recommendations and alternatives needed for informed decisions.

\section{Analytic Tree Construction Principles}

1. Use common and accepted graphic symbols for events, logic gates, and transfers. (See Figures 2, 3, 4, and 5. Figure 2 compares the "modified MORT" symbols recommended in this guide with "initial MORT" and Fault Tree Analysis symbols.)

2. Keep the analytic tree as simple as the complexity of the system allows (see Figures 6 and 7).

3. Keep the analytic tree logical and expect no miraculous occurrences. Use only those contributory events that are "necessary and sufficient" to produce the output event.

4. Select the logic gates and constraints (conditional events) that best describe true system functioning.

5. Select event titles or descriptions that are simple, clear, and concise. Avoid those that are abstract or are not readily understood by the intended users.

6. When constructing complex trees, limit the number of tiers on a single page to four or five (see Figure 8).

7. Use the Dewey decimal system for numbering events below the top event on the first page of the analytic tree. Locate the event identification above the upper right corner of the event symbol (see Figure 8).

8. Use a modified decimal system for identifying events below transfer symbols beginning with the letter designation of the transfer (see Figure 9). 


\begin{tabular}{|c|c|c|c|}
\hline Symbol Source & Modified MORT & Initial MORT & Fault Tree Analysis \\
\hline $\begin{array}{l}\text { Gate Output or } \\
\text { General Event }\end{array}$ & & $\perp$ & $T$ \\
\hline Base Event & & & \\
\hline $\begin{array}{l}\text { Undeveloped } \\
\text { Terminal Event }\end{array}$ & & & \\
\hline $\begin{array}{c}\text { Normally } \\
\text { Expected Event }\end{array}$ & & & \\
\hline Satisfactory Event & & & None \\
\hline AND Gate & & & \\
\hline OR Gate & & & \\
\hline Conditional Gate & & & \\
\hline Summation Gate & & None & \\
\hline Basic Transfer & $\mathbf{A}$ & & \\
\hline $\begin{array}{l}\text { Transfer from } \\
\text { Another Page }\end{array}$ & None & & \\
\hline $\begin{array}{c}\text { Assumed Risk } \\
\text { Transfer }\end{array}$ & & $R$ & None \\
\hline
\end{tabular}

Figure 2. Cross Index of Event and Logic Symbols. 


\section{Rectangle}

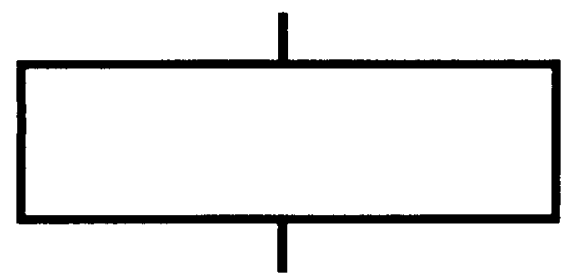

A general event or a gate output event resulting from the logical combination of contributory events acting through a logical gate.

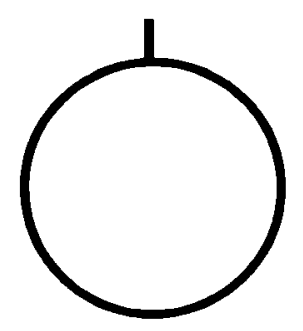

\section{Circle}

A base event requiring no further development. It is an independent event used only as a logic gate input.

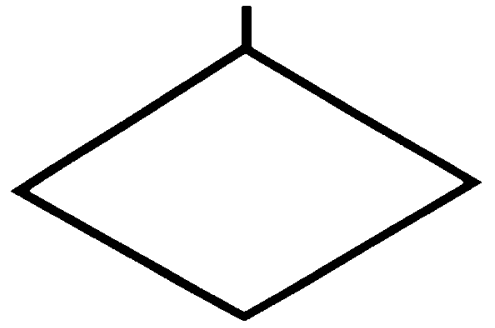

\section{Diamond}

An undeveloped terminal event not developed to its cause. Terminated for lack of information, resources or risks, or to avoid redundancy of analysis.

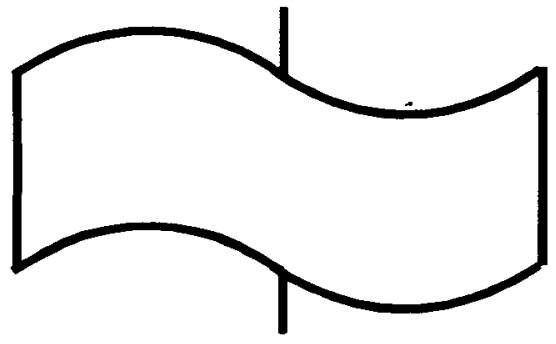

\section{Scroll}

A normally expected event that should occur naturally during normal functioning of the system.

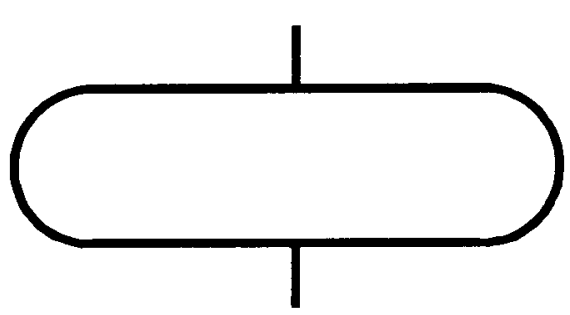

\section{Stretched Circle}

A satisfactory event that exists noncommittally in the system as a logic gate output and is used to show completion of a logical analysis.

Figure 3. Analytic Tree EVENT Symbols. 

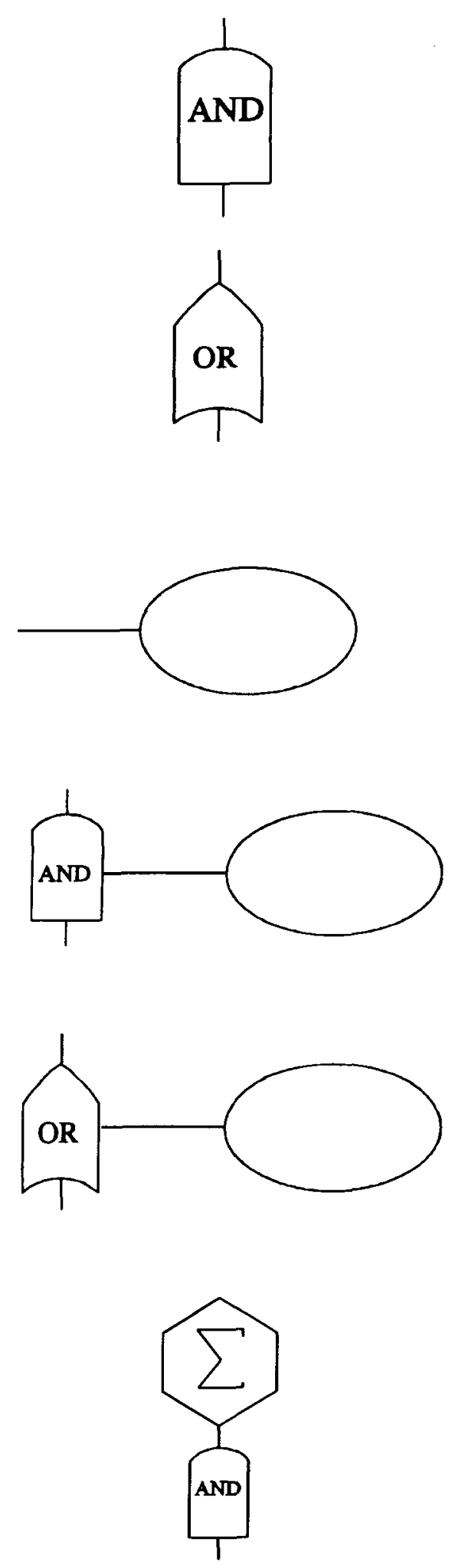

\section{AND Gate}

A logic gate that produces an output only when all input events occur. May contain the identifying word "AND".

\section{OR Gate}

Alogic gate that produces an output when one or more of the input events occur. May contain the identifying word "OR".

\section{CONSTRAINT}

A conditional erent that applies conditions or constraints to a basic logic gate or output event. Imposed condition is written in the ELLIPSE.

\section{CONDITIONAL AND Gate}

Input produces the output provided the conditions written in the ELLIPSE are satisfied. (Example: PRIORITY AND gate specifying order of input event occurrence.)

\section{CONDITIONAL OR Gate}

Input produces output provided the contsraint conditions are met. (Example: EXCLUSIVE OR gate enabling an output to occur only if a single input is present.)

\section{SUMMATION Gate}

A special logic gate which requires that an acceptable combination of input events be present to produce an output. Inputs can be present in varying proportions, as long as the sum of the inputs is adequate to generate an output.

Figure 4. Analytic Tree LOGIC GATE Symbols. 


\section{TRIANGLE}

The basic transfer operator represents the exact repetition of a tree section found elsewhere on the tree below an identical triangle.

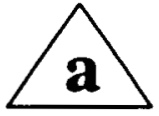

\section{Intrabranch TRANSFER}

Transfers substructure within a branch. Has identifying lower case letter.

\section{Interbranch/Interpage TRANSFER}

Transfers substructure from another branch or another page. Has an identifying capital letter.

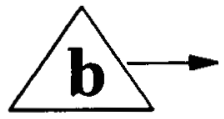

\section{OUT-TRANSFER, Same Page}

Horizontal arrow away from symbol shows transfer of substructure to another location on the same page in the direction the arrow points.

\section{IN-TRANSFER, Same Page}

Horizontal arrow toward symbol shows transfer from direction of arrow on same page.

\section{IN-TRANSFER, Other Page}

Vertical arrow toward base of symbol indicates transfer from branch on designated page.

\section{OUT-TRANSFER, Other Page}

"Recipient events" from other pages in broken lines above oversized triangle indicate transfer of substructure to recipient event locations on designated pages.

\section{SMALL OVAL}

Assumed risk transfer is used to transfer an assumed risk from any tree location to the assumed risk event (a SCROLL). The mumber of the assumed risk is indicated inside the symbol as shown. It normally originates as a DIAMOND (undeveloped terminal event).

Figure 5. Analytic Tree TRANSFER Symbols . 

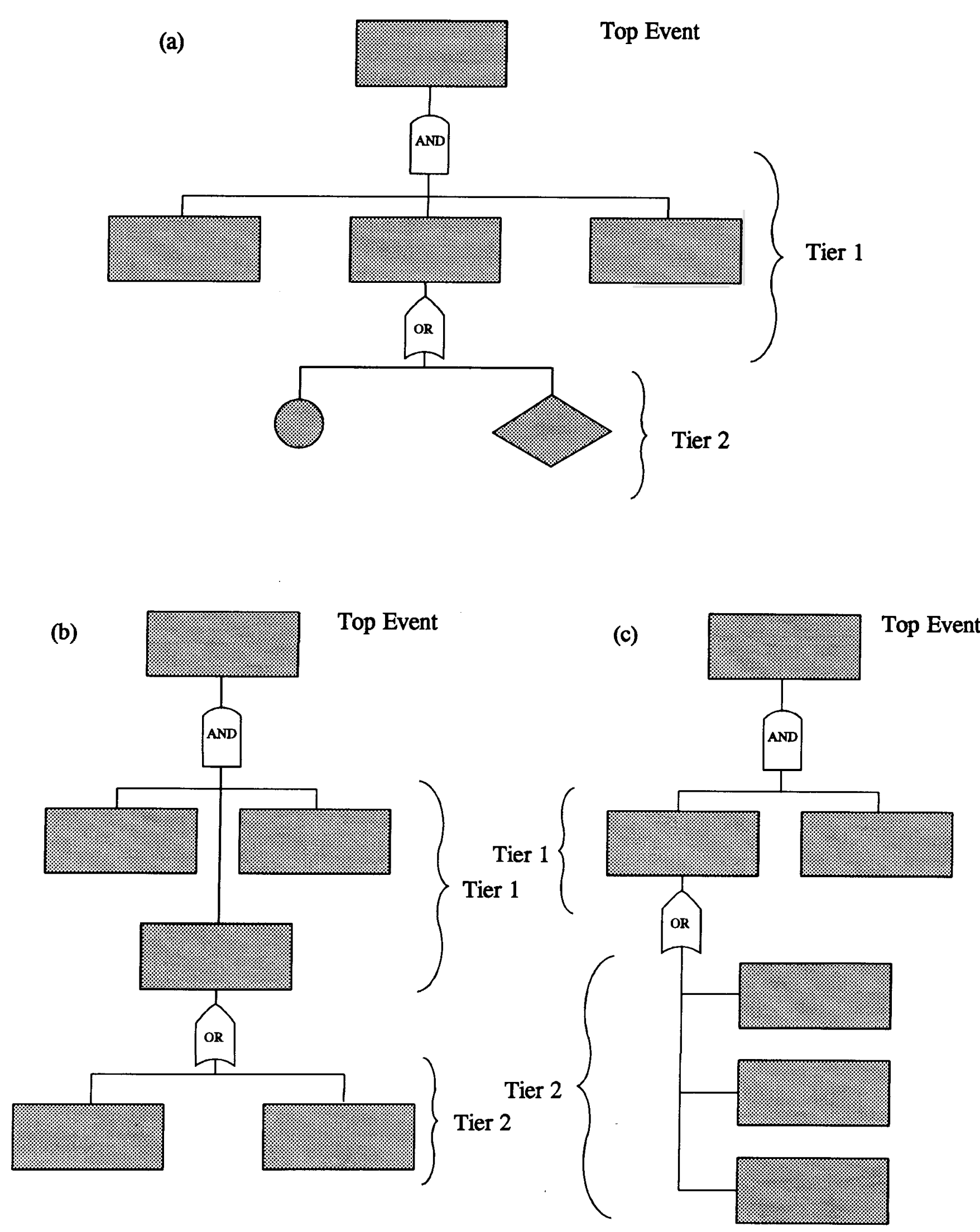

Figure 6. Acceptable Tier Arrangements. 


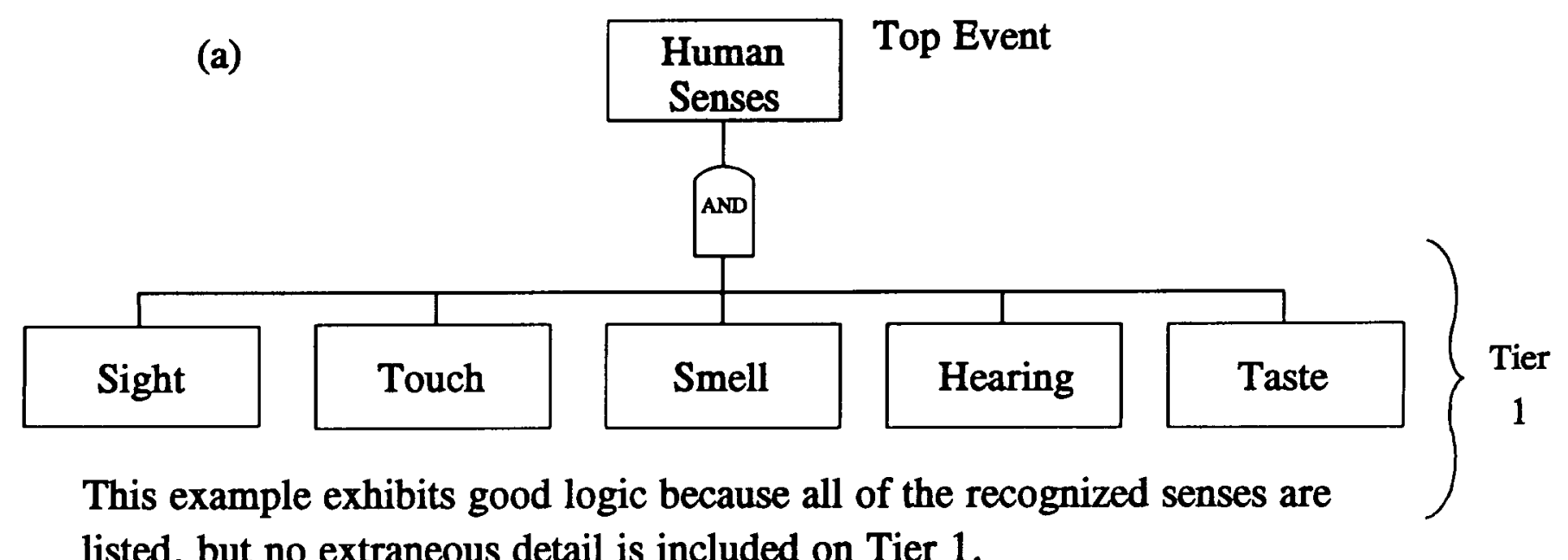
listed, but no extraneous detail is included on Tier 1 .

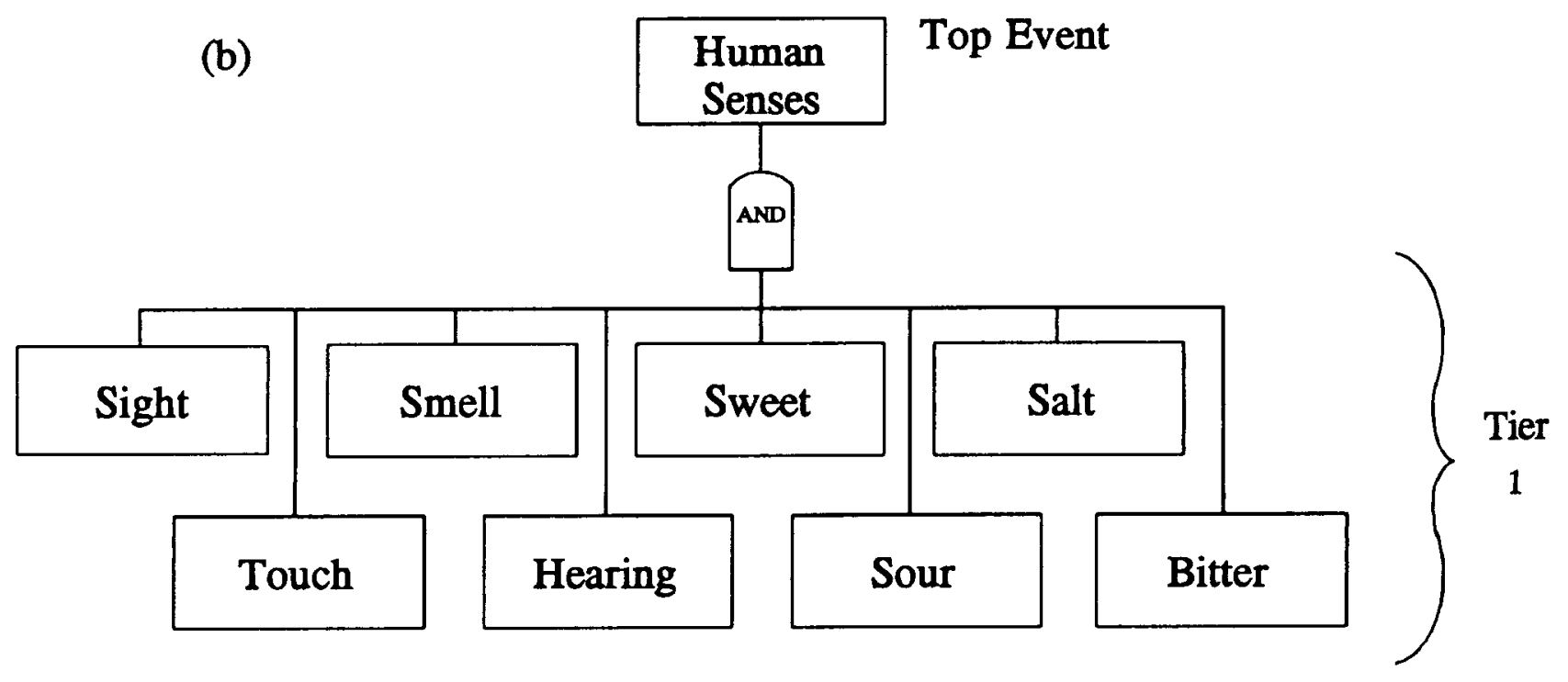

Poor logic has been used here by listing the detailed constituents of taste on the same tier as the other four senses. If this level of detail is desired, the contributory events should be listed on Tier 2 under the appropriate sense.

Figure 7. Examples of Good and Poor Logic. 


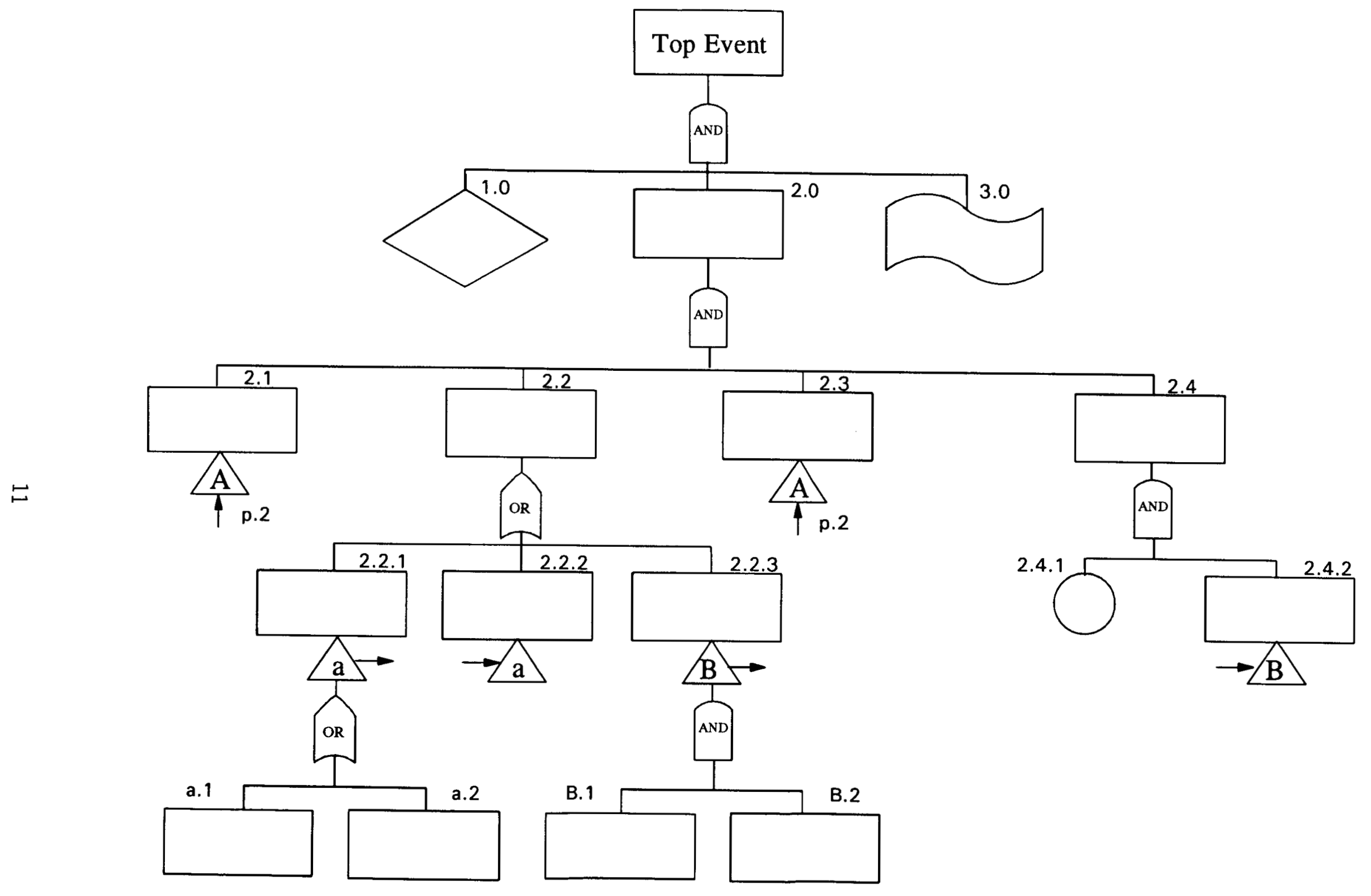

Figure 8. Sample Analytic Tree - Sheet 1. 


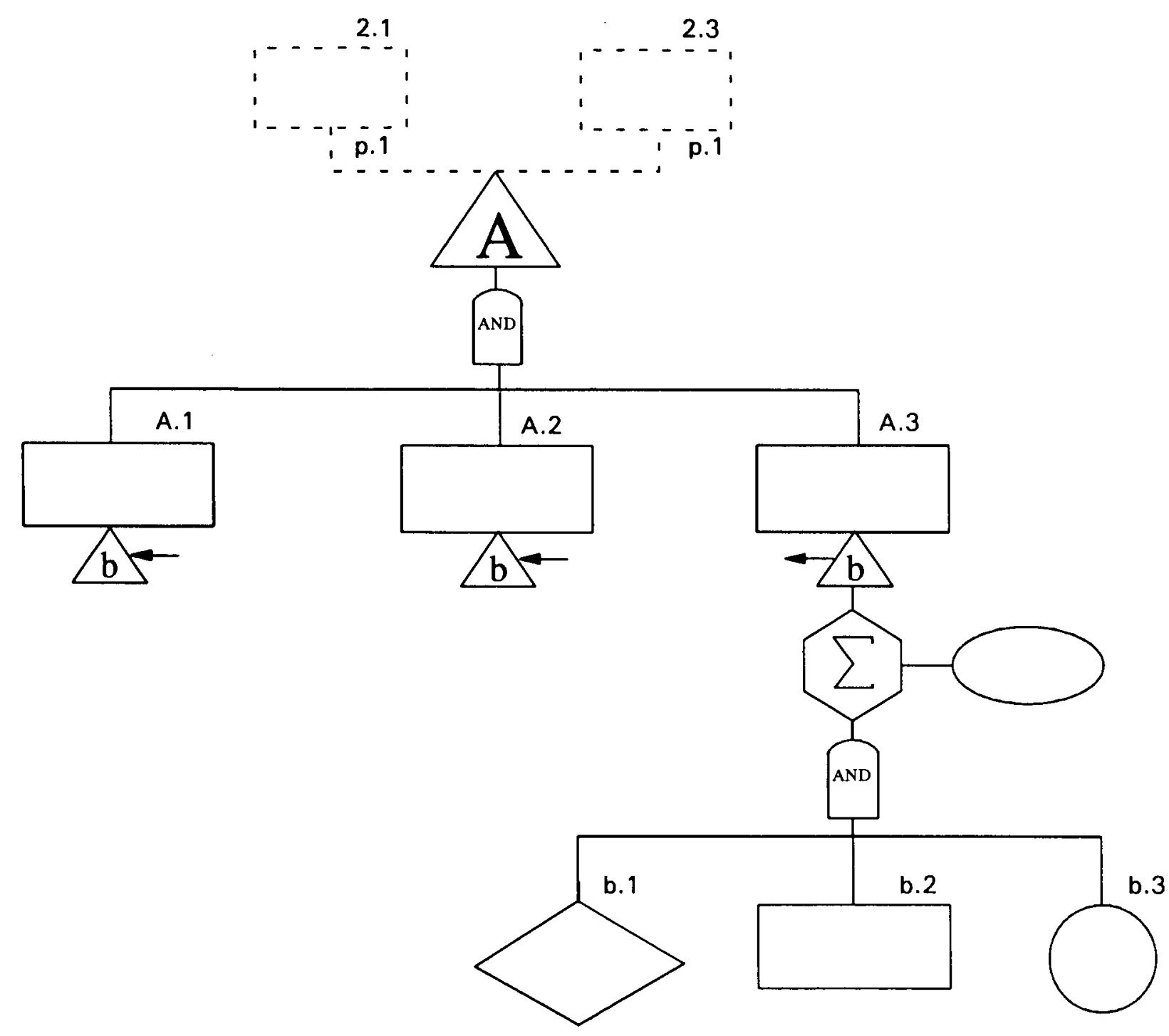

Figure 9. Sample Analytic Tree - Sheet 2. 
9. Use transfers to avoid duplication of identical branches or segments of the tree and to reduce single-page-tree complexity (see Figures 8 and 9).

a. Identify "intrabranch" transfers by lowercase letter designations and "interbranch" or "interpage" transfers by capital letter designations, i.e., $\triangle$ and $\underset{\mathrm{A}}{\mathrm{A}}$, respectively.

b. Show transfers into a branch of the analytic tree by an arrow pointing toward the transfer symbol from the general direction of transfer; horizontal for transfers on the same page and vertical for those from another page (see Figures 8 and 9).

c. Show transfers out of a branch to another location on the same page by an arrow pointing away from the transfer symbol in the direction of transfer (see Figures 8 and 9).

d. For transfer of a branch to another page, list the "recipient events" in broken lines above an "oversized" transfer symbol on the page where the transfer originates. The page to which the substructure is to be transferred is specified below the lower right corner of the recipient event. A similar notation at the transfer symbol on the receiving page shows the origin page of the transferred branch (see Figures 8 and 9 , transfer "A").

10. Do not number or letter logic gates. They are adequately identified by the input events that operate through them and the resulting output events.

11. Follow the convention of indicating order of performance or time sequencing from left-to-right, for related events on a single tier. 


\section{ANALYSIS STEPS}

The basic steps in analyzing any system through use of an analytic tree include:

1. Define the top event. That which you desire to achieve, or which you desire to prevent from happening is the top event. Make it as specific as you can so that contributory events can be clearly and accurately recognized and defined.

2. Acquire a comprehensive understanding of the management system or safety system to be analyzed. Only by fully understanding the system, its constituents and details, and their interrelationships and interfaces can a logical and complete analysis be performed, which identifies and considers all events which are necessary and sufficient to produce the top event or outcome. It is often necessary and desirable, particularly with success trees, to:

- $\quad$ Define the "ideal" management system or safety system

- Compare the existing system with it

- Incorporate those events and logic gates in the analytic tree that are required to bring the present system up to the ideal.

3. Construct the analytic tree. With the top event defined and a thorough understanding of the system acquired, the analyst then constructs the analytic tree, using appropriate logic gates and standardized event symbols and transfers. Specifics of tree construction will be discussed later.

Proper logic is followed to ensure that all events meet the "necessary and sufficient" criterion, i.e., those events are specified which are the minimum necessary, and no more than is sufficient, to immediately produce the logic gate output event. Each event is essential to the tree logic (necessary) and no other information is needed (sufficient) to achieve the stated output. All other events are either excluded as being extraneous or are relegated to a supportive and more detailed lower tier.

Steps 2 and 3 ordinarily are not separate and distinct as suggested here, but additional information about the system is often discovered and applied as tree construction develops.

4. Validate the analytic tree. Once construction of the analytic tree has been completed, one or more knowledgeable persons should review the tree events and logic for accuracy and completeness - for omissions and oversights. The purpose of this validation review is to confirm that:

- The tree meets its intended objectives

- The system and its functioning are fully and clearly described

- Inputs to logic gates are necessary and sufficient to logically produce the stated output events.

Validation by other persons has proven time and again to be essential to produce useful, error-free analytic trees, which identify system weaknesses and strengths and lead to proper assessment of identifiable risks. Often, consultation with others to validate the tree begins before construction is complete, so steps 3 and 4 sometimes overlap. 
5. Evaluate the analytic tree. Following validation of the tree, it is evaluated to identify critical paths to achievement of the top event. Thorough study of the elements and interrelationships in the tree will enable the analyst to identify oversights and omissions in the safety/management system, and to ensure that identifiable risks are presented to the proper management levels for acceptance or resolution. As the analyst evaluates the failure or success paths from base events to the top event, paths or chains of varying importance and system impact will emerge. The relevance to system output of these paths, and the events of which they are composed, must be carefully weighed and major emphasis placed on those of greatest significance. (This, of course, will require value judgments by the analyst, but they should be based realistically on what the analysis reveals.) Management must not only be informed of the risks involved, but also of their relative significance, if they are to make the best risk-related decisions.

6. Conduct tradeoff studies. Tradeoff, cost-benefit, or risk-benefit studies are needed to determine which risks should be assumed, which risks cannot be assumed, and where controls can most effectively be applied to achieve the desired objective or prevent the undesired occurrence.

7. Management makes rational and informed decisions concerning the safety/management system. The results of analytic tree evaluation and subsequent tradeoff studies lead logically to recommendations and alternative solutions, from which management can make knowledgeable and informed decisions on system control and repair and on risk assumption. 


\section{TREE CONSTRUCTION}

Analytic tree construction is a logical development of the top event, using deductive reasoning to progress through successively more specific events to basic events or causes, from which sequential chains of success or failure begin. The various levels of tree development are tiers and branches of contributory events that are sequentially linked by logic gates. Each tier of tree development contains those events which, when processed through the logic gate, are necessary and sufficient to lead directly to the success or failure of the event on the next higher tier. Branching occurs when any of the multiple events, which may operate through a logic gate to produce a common higher event, have substructures of their own.

\section{Basic Principles}

Use of the standardized approach for analytic tree construction and identification requires adherence to 11 basic principles.

\section{Symbols}

Use common and accepted graphic symbols. Analytic tree symbols may be categorized into three groups: (a) events, (b) logic gates, and (c) transfers. Although an DOE approved MORT template currently does not exist, logic symbol templates that can aid the analyst in tree construction are available from several commercial sources.

Events. An event is a possible condition or state of a system element or function. It may be a long-lived condition, or it may arise spontaneously or gradually from a dynamic change of state. If it results in a desirable or intended occurrence, it is a success or normal event. If it results in system degradation or failure, or an abnormal occurrence, it is a failure or fault event. The same common symbols are used as components of both success trees and fault trees.

Event symbols are of five basic types, each of which represents a different kind of event (see Figure 3).

1. The RECTANGLE is the general event symbol and is used extensively in all trees, but particularly in success trees. It is also used to represent a gate output event, resulting from the logical operation of contributory events acting through a logic gate.

2. The CIRCLE represents a base event that requires no further development. It is an independent event that defines an inherent system element fault or a base-level success, and is used only as an input to a logic gate.

3. The DIAMOND represents an undeveloped terminal event, which is not developed further because of:

a. Low relevance or low risk, i.e., a JSA (Job Safety Analysis) is not performed on a particular task, because it has a low potential for accident; and the low risk is assumed.

b. Lack of adequate information or resources for solution, i.e., the nearest hospital is $4 \mathrm{O}$ miles from the work site, and cost is prohibitive to move nearer; therefore, event development is terminated and the distance risk is assumed.

c. Redundancy avoidance when another analytic tree gives the needed information, i.e., in developing a detailed use readiness tree for a new complex facility, the safety criteria event should be a DIAMOND, and reference made to the "Occupancy-Use 
Readiness Manual - Safety Considerations", where the safety criteria tree is already developed.

d. Influence on the scope of the tree, but not necessary to the development of the analytical logic, i.e., safety considerations only are analyzed in the Occupancy Use Readiness Tree; but undeveloped interfacing events with other organizations are shown because they influence tree scope, even though they are not necessary to logic development.

In cases (a) and (b), the event logically becomes an "assumed risk." In case (c), a footnoted reference identifies the supplementary analysis. In case (d), "fringe area" events are identified that do not contribute significantly to development of the final system outcome, but which should be included on the tree to indicate that their influence on the extent or depth of system analysis has been considered.

Like base event circles, terminal event diamonds represent base-level events which are used only as logic gate inputs.

4. The SCROLL represents a normally expected event, which is expected to occur naturally during normal functioning of the system. It has the same meaning as the "house" in Fault Tree Analysis symbolism. Assumption of some risks in any major operation is normally expected, so the "assumed risk" event would be a SCROLL. Likewise, in assessing deviations in personnel performance, normal variability among workers would be expected and should be depicted as a SCROLL.

5. The STRETCHED CIRCLE is a satisfactory event, which simply exists in the system but is neither fault-oriented nor success-oriented. It is a logic gate output event that is most often used to show completion of logical analysis. It covers such events as the presence of personnel or objects in an energy channel because they are needed there to perform a functional task.

Logic Gates. A logic gate performs a discrete operation upon contributory events to produce a logical output event. The fundamental logic gates for analytic tree construction are the AND gate and the OR gate. Many analytic trees can be constructed using only these basic logic gates. If a CONSTRAINT symbol is added to a basic logic gate to modify it or impose special conditions on its operation, greater flexibility is added. Further addition of a SUMMATION gate should provide the analyst with all the logic gates he needs for thorough analysis of the system (see Figure 4).

The AND gate produces an output only if all required input events coexist. In other words, all contributory events must occur for an AND gate to produce an output event. The OR gate produces an output event when one or more of the contributory events occur.

The addition of a CONSTRAINT symbol (an ELLIPSE) to the side of a basic logic gate applies conditions or constraints to the basic gate to create a CONDITIONAL gate, which inhibits or prevents an output until the specified condition is met. Typical of a CONDITIONAL gate are the PRIORITY AND gate, which requires a particular input event sequence to cause occurrence of the output event, and the EXCLUSIVE OR gate, which enables the output event to occur, if one, and only one, of the input events is present.

The SUMMATION gate is a special logic gate that requires that an acceptable combination of the input events occurs to produce an output. This allows for contributory events to be present in varying proportions, as long as the composite contribution is sufficient to produce occurrence of the output event; that is, a deficiency in one or more input events can be compensated by greater contributions of the other input events. Unlike the Fault Tree Analysis "basic and/or" gate logic, which requires each input event to be either present or absent (a strict binary logic), the 
SUMMATION gate allows any contributory event to be present to any degree from $O$ to $100 \%$, as long as the total input from all events can generate the output event. For example, in the Behavioral Change Tree, four input events operate through a SUMMATION gate to produce the output event, "Select Means for Introducing Behavioral Change." Deficiencies in input 1, "Control Selection and Placement," can be compensated by improved performance on input events 2, 3, and 4, "Control Training," "Control Factors That Influence Attitudes," and "Control Organizational Psychology Factors," respectively. This provides a satisfactory combination of events for introducing the desired behavioral change.

Transfers. A TRANSFER symbol indicates that an event, a series of events, or a complete branch of the analytic tree is transferred from one location on the tree to another. Rather than duplicating that portion of the tree in the second location, a transfer TRIANGLE is used to indicate an exact repetition of that tree section at the second, third, etc., location. Use of a special SMALL OVAL to represent transfer of an "assumed risk" event is peculiar to MORT. Both TRANSFER symbols, the tree-section transfer TRIANGLE, and the "assumed risk" transfer SMALL OVAL are used to avoid repetition, conserve space, and simplify tree construction (see Figure 5). Additional information on use of TRANSFER symbols will be provided as other tree construction principles are discussed.

\section{Simplicity}

Keep the analytic tree as simple as the complexity of the system allows. When the analyst has gained a thorough understanding of the system to be analyzed, he begins to lay down its logical progression from the top event to base events. He should be ready to get additional system information as the tree reveals that need. However, he should be selective in determining the depth of analysis and should use the undeveloped termination event DIAMOND when further development is clearly not justified. This usually occurs when all the relevant dependencies and the necessary and sufficient input events have been identified. Normally, the analyst will clean up and further simplify the analytic tree that he has used as an analysis tool, before presenting it to management for use in making risk assumption and risk resolution decisions.

\section{Logic}

Keep the tree logical and expect no miraculous occurrences. Deductive analysis, using an analytic tree as a logic aid, should proceed logically from the top event to base events. Related events at the same level of logic and detail are entered on a single tier and are joined by a line before being processed through a logic gate. A vertical line and a logic gate join a gate output event on one tier with its more detailed contributory events on the next lower tier. Ideally, all events on the same tier will be on the same horizontal level [Figure 6(a)]; however, because of space limitations and page-fitting problems during tree construction, they are often joined to a common horizontal line by vertical extensions of varying lengths [Figure $6(\mathrm{~b})$ ], or they are joined to a single vertical line and listed ladder-like, one below the other [Figure 6(c)]. Any of the tier arrangements in Figure 6 are acceptable. Figure 7 displays examples of good and poor logic in placement of events in analytic tree tiers.

Expect no miracles, either good or bad. The analytic tree cannot do the work for the analyst. It is simply a tool for use in organizing and systematizing thinking to aid one in finding the right answers. Its output can be no better than the quality and organization of its inputs. Be reasonable, logical, and practical, and expect logical and rational sequences to develop through logic gates, as identifiable events interrelate and interact. Lower tier input events should be only those that are necessary and sufficient to produce the gate output event.

Do not struggle to develop outlandish or irrational events that would require a miracle for occurrence, such as postulating simultaneous occurrence of a building fire, bomb threat, severe winter 
storm, tornado, earthquake, and an impending nuclear attack in analyzing an Emergency Response System.

\section{Gate Selection}

Select the logic gates and constraints (conditional events) that best describe true system functioning. The basic AND, OR, and SUMMATION logic gates, modified as necessary by CONSTRAINTS, provide sufficient flexibility to accurately describe the logical processing of contributory input events and to produce the defined output events at each level of the analytic tree. Proper selection and use of logic gates will establish a logical progression of identifiable event interactions through the tree to tie the top event to its root contributors at the base event level and to accurately describe the way the system really works.

\section{Event Titles}

Select event descriptions that are simple, clear, and concise. Event descriptions should be sufficiently descriptive and understandable that the analytic tree user can grasp their meaning and follow the analytic process without having to refer to explanatory data found elsewhere. Analysts should particularly avoid event descriptions that are abstract, or which contain terms with which the intended user is unfamiliar. Additionally, event descriptions for systems with considerable people involvement should include active verbs ("do" verbs), such as "plan," "prepare," "control," "implement," etc., to convey the precise nature of input events that are necessary and sufficient to generate the output events and, ultimately, the top event.

\section{Tier Limits}

When constructing complex trees, limit the number of tiers on a single page to four or five. There are two basic reasons for imposing such a limitation:

1. Most trees are reproduced on $8-1 / 2 \times 11$ or on $11 \times 17$ in. sheets for inclusion in a document or for convenient use on the job. The complexity of branches of the analytic tree will cause some variance in the number of tiers that may appear on a single page; but normally, more than four or five tiers cannot be reproduced legibly or read without magnification.

2. Use of the Dewey decimal system for event identification (discussed in principle 7) becomes cumbersome and difficult to manage beyond five digits (five tiers). A modified decimal system restores the simplicity of event identification below TRANSFER symbols (see principle 8).

\section{Event Identification}

Use the Dewey decimal system for numbering events below the top event on the first page of the analytic tree. Each event is uniquely identified by a Dewey decimal number located above the upper right corner of the event symbol. The number of nonzero digits in the Dewey decimal event numbering system corresponds to the tier on which the event is located, i.e, the third tier contains 3 digit event numbers. Table 1 gives representative Dewey decimal numbers for various tiers.

A Dewey decimal event identification number not only uniquely describes an event but also systematically traces its development through subbranches and branches to its progenitor event on the first tier. Each successively higher level event can be identified by dropping the last digit from the number as shown below: 


\section{Table 1}

\section{Dewey Decimal Event Identification Numbers}

\begin{tabular}{|c|c|}
\hline Tier & Number Designation \\
\hline Top Event & Unnumbered \\
\hline First & $1.0,2.0,3.0,4.0 . . . n .0$ \\
\hline Second & $1.1,1.2,1.3, \ldots .2 .1,2.2,2.3 . . . n . m$ \\
\hline Third & 1.1.1, 1.1.2, 1.1.3...2.1.1, 2.1.2...n.m.p \\
\hline Fourth & 1.1.1.1, 1.1.1.2...2.1.3.1, 2.1.3.2...n.m.p.q. \\
\hline Fifth & 1.1.3.2.1, 1.1.3.2.2...2.1.4.2.1...n.m.p.q.r \\
\hline
\end{tabular}


Top Event
1.0
1.1
1.1.1
1.1.1.1
1.1.1.1.1
First Tier
Second Tier
Third Tier
Fourth Tier
Fifth Tier

Figure 10 shows another numeric progression in tree format. (See also Figure 8.)

\section{Modified Event Identification}

Use a modified decimal system for numbering events below transfer symbols beginning with the letter designation of the transfer, i.e., A.1.3.2 or a.l.3. Numbering progresses through succeeding subtiers in the same way as the pure numerical Dewey decimal system, as shown in Table 2 and the accompanying examples.

Alphanumeric progression from the fourth subtier to the TRANSFER is shown below:

$\begin{array}{ll}\text { D } & \text { TRANSFER } \\ \text { D.2 } & \text { First Subtier } \\ \text { D.2.2 } & \text { Second Subtier } \\ \text { D.2.2.1 } & \text { Third Subtier } \\ \text { D.2.2.1.2 } & \text { Fourth Subtier }\end{array}$

Figure 11 shows the same progression in tree format. (See also Figure 9.)

\section{Transfer Use}

Use transfers to avoid duplication of identical branches or segments of the tree and to reduce single page tree complexity. Whenever two or more gate output events have identical details in the substructures contributing to their occurrence, that substructure should be constructed under only one of the output events, and then transferred to the others through the use of TRANSFER symbols (see Figure 8, TRANSFERS "a" and "B"). Be careful to avoid the inclination to force a substructure that "almost fits," but has basic differences, into a "transferable" structure. TRANSFERS should be used also below the bottom tier events on a page to indicate continuance of subbranches of those events on other pages. Additionally, whenever there is insufficient space on a page to develop a branch below an event at any level, a TRANSFER immediately below that event indicates that the branch is developed on another page (see Figures 8 and 9, TRANSFER "A").

Transfer Identification. Identify "intrabranch" transfers by lowercase letter designations, and "interbranch" or "interpage" transfers by capital letter designations, i.e., $\mathbb{A}$ and $\mathbb{A}$, beginning with "a" and "A," respectively, and proceeding logically. Refer to Figures 7 and 8 where TRANSFERS "a" and " $b$ " transfer substructures within a branch are intrabranch transfers; TRANSFER " $A$ " is the transfer of a branch between pages (interpage transfer), and TRANSFER " $B$ " is a substructure transfer from one branch to another (interbranch transfer).

Interpage Transfers. Show transfers into a branch of the analytic tree by an arrow pointing toward the transfer symbol. For transfers from a branch on the same page, the arrow points to the side of the TRANSFER symbol from the direction in which the transfer is made, i.e., $\mathrm{a}$ - (see Figures 8 and 9, TRANSFERS "a," "b," and "B"). For transfers from another page, the arrow points to the base of the TRANSFER triangle, and the source page is listed adjacent to the arrow, i.e., (see Figure 8, TRANSFER "A").

Transfers In. Show transfers out of a branch to another location on the same page by an arrow pointing away from the transfer symbol in the direction of the transfer, i.e. Bu- (See Figures 8 


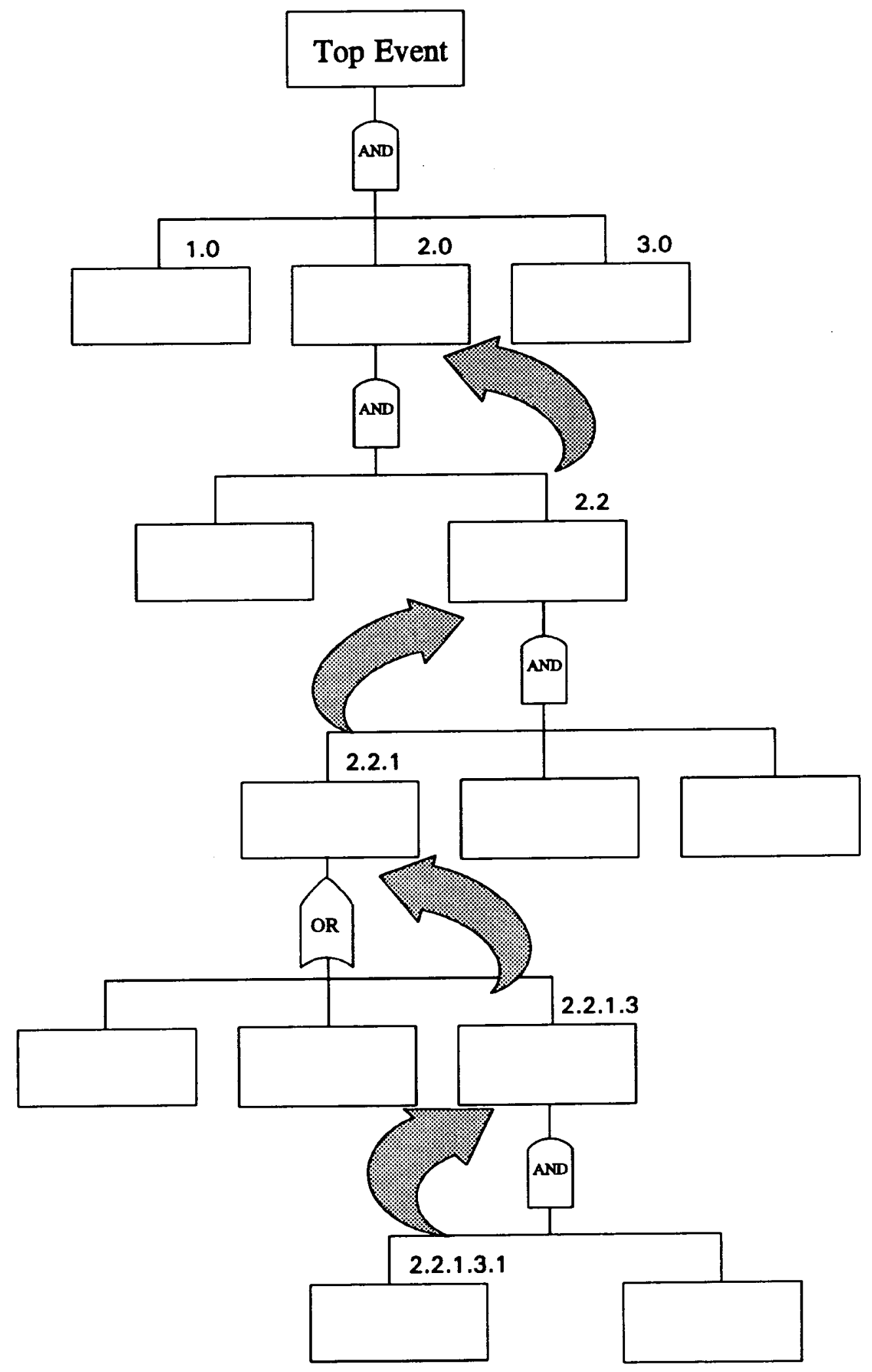

Figure 10. Another Numeric Progression in Tree Format. (Also see Figure 8.) 


\section{Table 2 \\ Modified Dewey Decimal Event Identification Numbers}

\begin{tabular}{|l|c|}
\hline $\begin{array}{c}\text { Transfer } \\
\text { Subtier }\end{array}$ & Number Designation \\
\hline Transfer & A, B, C...N \\
First & A.1, A.2, A.3...N.m \\
Second & A.1.1, A.1.2..A.2.1, A.2.2...N.m.p \\
Third & A.1.1.1, A.1.1.2, A.1.1.3...N.m.p.q \\
\hline
\end{tabular}




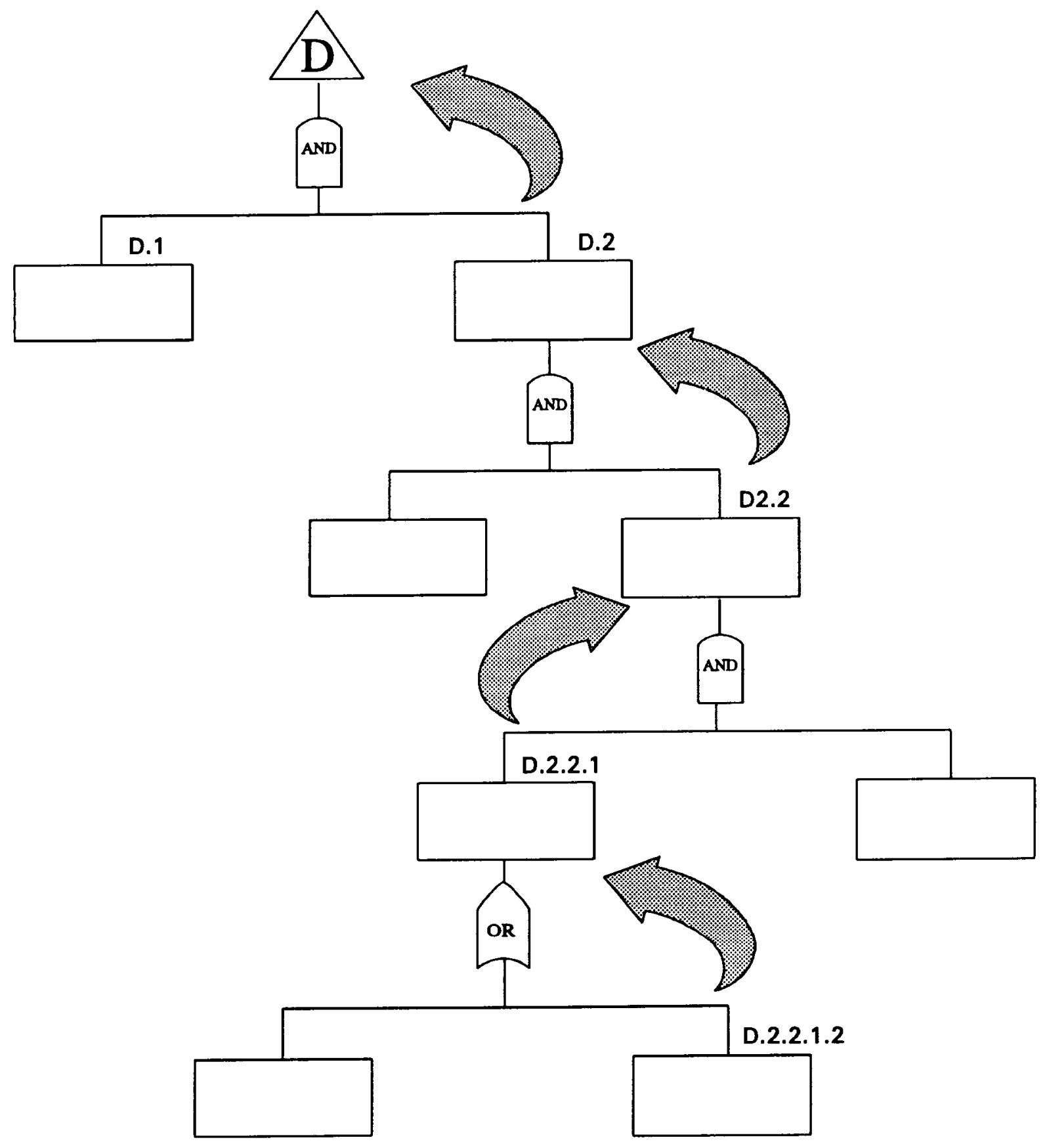

Figure 11. Alphanumeric Progression from the Fourth Subtier to the TRANSFER in Tree Format. (Also see Figure 9.) 
and 9, TRANSFERS "a," "b," and "B.") Remember, transfers out are away from the symbol and transfers in are toward the symbol; also, arrows point in the general direction of the transfer, horizontal for transfers on the same page and vertical for transfers from another page.

Intrapage Transfers. For transfer of a branch to another page, list the "recipient events" in "broken" lines above an "over-sized" transfer symbol on the page where the transfer originates. Also, specify the page or pages to which the branch will be transferred by listing the appropriate page number below the lower right corner of the recipient event. (See Figure 9, TRANSFER "A". Note that the " $\mathrm{A}$ " structure transfers to two locations on page 1 , event 2.1 and event 2.3.) On a multipage tree, it is conceivable that there could be several recipient events fed by a single transfer structure, and that they might be located on different pages, i.e., one recipient event on page 3 , another on page 4 , still another on page 6 , etc. In such a case, all the recipient events should be identified by "broken lines" (dashed lines) above the TRANSFER triangle on the transfer originating page, and each recipient event should be further identified by its page location, i.e., p. 3, p. 4, p. 6, etc. When a multi-page analytic tree with many transfers is compiled onto a single, oversized "fold sheet," such as the universal MORT diagram, the page designations of transfer and recipient event locations can be replaced by coordinate area designations. The coordinate system could be based on an unlined cartesian grid with a numbered ordinate (vertical) and a lettered abscissa (horizontal) to give such event locations as $1 \mathrm{a}, 4 \mathrm{c}, 7 \mathrm{f}$, etc.

\section{Gate Identification}

Do not number or letter logic gates; use numeric and alphanumeric decimal identification designations only for events. Logic gates are defined by the input events that operate through them and the specific output events that occur. Therefore, it is not necessary to assign specific identification numbers to logic gates; for they are fully defined by the events they serve.

\section{Intratier Priorities}

Follow the convention of indicating time sequencing or order of performance from left to right for related events on a single tier. It should also be apparent that a higher tier event has greater significance (more impact on the top event) and occurs later than the more detailed contributory events located on lower tiers within its branch.

Construction and use of an analytic tree involves deductive analysis beginning with a stated top event, and then proceeding through the immediate causal events, intermediate events, and detailed events to the base events, which originate the sequential chains that lead to top event occurrence. If the analyst has done his or her job well, the events will occur in the logical sequence displayed on the analytic tree. Critical paths to success or failure can then be discerned by the user, and appropriate fixes can be applied at the proper event level to ensure the desired success or prevent the predicted failure.

The user's task will be made simpler, more logical, and more orderly if he or she knows that the analytic tree author has also established a left-to-right sequencing of occurrence among events on a single tier. The analytic tree is a tool for use in system analysis; anything that contributes greater simplicity, order, and logic to it enhances its usefulness. 


\section{BIBLIOGRAPHY}

Johnson, W. G., MORT - The Management Oversight and Risk Tree, SAN 821-2, February 12, 1973.

Knox, N. W., and R. W. Eicher, MORT User's Manual, ERDA-76/45-4, SSDC-4 (Rev. 2), February 1992.

Lambert, H. E., System Safety Analysis and Fault Tree Analysis, UCID-16238, May 9, 1973.

Nertney, R. J., and J. A. Buys, Training As Related to Behavioral Change, ERDA-76-45-6, SSDC-6, June 1976.

Nertney, R. J., J. L. Clark, and R. W. Eicher, Occupancy-Use Readiness Manual - Safety Considerations, ERDA-76-45-1, SSDC-1, September 1975. 


\section{COMPLETED SSDC PUBLICATIONS}

SSDC-1

SSDC-2

SSDC-3

SSDC-4

SSDC-5

SSDC-6

SSDC-7B

SSDC-8

SSDC-9

SSDC-10

SSDC-11

SSDC-12

SSDC-13

SSDC-14

SSDC-15

SSDC-16

SSDC-17

SSDC-18

SSDC-19

SSDC-2O

SSDC-21

SSDC-22

SSDC-23

SSDC-24

SSDC-25

SSDC-26

26.1

26.2

26.3

26.4

26.5

26.6

26.7

26.8

26.9

26.10

26.11

26.12

26.13

26.14

26.15

26.16

26.17

26.18

SSDC-27

SSDC-28

SSDC-29

SSDC-30

SSDC-31
Occupancy-Use Readiness Manual

Human Factors in Design

A Contractor Guide to Advance Preparation for Accident Investigation

MORT User's Manual

Reported Significant Observation (RSO) Studies

Training as Related to Behavioral Change

DOE Guide to the Classification of Recordable Accidents

Standardization Guide for Construction and Use of MORT-Type Analytic Trees

Safety Information System Guide

Safety Information System Cataloging

Risk Management Guide

Safety Considerations in Evaluation of Maintenance Programs

Management Factors in Accident/Incidents (Including Management Self-Evaluation Checksheets)

Events and Causal Factors Charting

Work Process Control Guide

SPR0 Drilling and Completion Operations

Applications of MORT to Review of Safety Analyses

Safety Performance Measurement System

Job Safety Analysis

Management Evaluation and Control of Release of Hazardous Materials

Change Control and Analysis

Reliability and Fault Tree Analysis Guide

Safety Appraisal Guide

Safety Assurance System Summary (SASS) Manual for Appraisal

Effective Safety Review

Construction Safety Monographs

Excavation

Scaffolding

Steel Erection

Electrical

Housekeeping

Welding/Cutting

Confined Spaces

Heating of Work Spaces

Use of Explosives

Medical Services

Sanitation

Ladders

Painting/Special Coatings

Fire Protection

Project Layout

Emergency Action Plans

Heavy Equipment

Air Quality

Accident/Incident Investigation Manual (2nd Edition)

Glossary of SSDC Terms and Acronyms

Barrier Analysis

Human Factors Management

The Process of Task Analysis
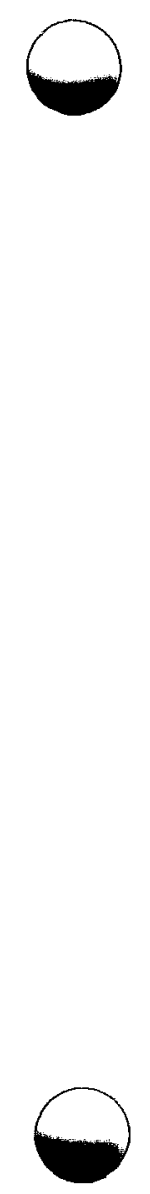
SSDC-32

SSDC-33

SSDC-34

SSDC-35

SSDC-36

SSDC-37

SSDC-38

SSDC-39

SSDC-4O

SSDC-41
The Impact of the Human on System Safety Analysis

The MORT Program and the Safety Performance Measurement System

Basic Human Factors Considerations

A Guide for the Evaluation of Displays

MORT-Based Safety Professional/Program Development and Improvement

Time/Loss Analysis

Safety Considerations for Security Programs

Process Operational Readiness and Operational Readiness Follow-On

The Assessment of Behavioral Climate

Investigating and Reporting Accidents Effectively 